\title{
Broad view on hard X-ray background emission of the Galaxy
}

\author{
Roman Krivonos $^{1,2}$, Mikhail Revnivtsev ${ }^{2,3}$, Sergey Tsygankov ${ }^{1,2}$, \\ Eugene Churazov ${ }^{1,2}$ and Rashid Sunyaev ${ }^{1,2}$ \\ ${ }^{1}$ Max Planck Institute for Astrophysics, Garching, Germany \\ email: krivonos@mpa-garching.mpg.de, \\ ${ }^{2}$ Space Research Institute, Moscow, Russia \\ ${ }^{3}$ Excellence Cluster Universe, Technische Universitat Muenchen, Garching, Germany
}

\begin{abstract}
The nature of the Galactic Ridge X-Ray Emission (GRXE) has been under scientific debate since its discovery more than 30 years ago. It is observed as extended emission along the Galactic disk. The question was: is GRXE truly diffuse or is it composed from a large number of unresolved point sources? Using near-infrared Galaxy maps measured with the DIRBE experiment and data from the INTEGRAL observatory, we show that the galactic background in the energy range $20-60 \mathrm{keV}$ originates from the stellar population of the Galaxy, which is in contrast to the diffuse nature believed before (Krivonos et al., 2007). Here we show preliminary results of studying the transition region from hard X-rays to gamma diffuse background of the Galaxy, revealing the broad band picture of Galactic Background emission.
\end{abstract}

Keywords. Galaxy: disk,Galaxy: bulge,Galaxy: stellar content,ISM: cosmic rays

In many high-energy and $\mathrm{MeV}$ - observations of the Galactic Plane the gamma-ray continuum emission was detected (see e.g. Kraushaar et al., 1972) and is believed to originate from the interaction of cosmic rays with the interstellar medium. This suggests that at energies $>100-200 \mathrm{keV}$ a change in the nature of the unresolved Galactic emission to cosmic-ray induced background should occur. This implies detection of the rising part of the Ridge spectrum in the transition region. Indications for this were found in INTEGRAL/SPI measurements (e.g. Bouchet et al., 2008).

To investigate the Galactic background in the transition region between compact objects in X-rays, and truly diffuse (cosmic ray induced) in the $\mathrm{MeV}$ energy band, additional observations were scheduled of the Galactic disk $\left(l=-45^{\circ}\right)$. Here we report on the possible detection of the rising part of the Galactic background emission spectrum above 100 $\mathrm{keV}$ measured with the IBIS/ISGRI instrument onboard the INTEGRAL observatory. The spectrum's slope of 1.5 is compatible with that expected for the spectrum of cosmicray induced background emission and/or positron annihilation continuum. Subtracting a model of the high-energy rising part from the measured spectrum we obtained a GRXE spectrum at lower energies which is in good agreement with that measured in the Galactic center. This implies that the source population responsible for GRXE production is similar in the Galactic center and the plane in 17-100 keV energy band.

\section{References}

Krivonos R., Revnivtsev M., et al., 2007, A\&A 463, 957

Kraushaar et al., 1972, ApJ, 177, 341

Bouchet et al., 2008, The Astrophysical Journal, 679, 1315 